\title{
Sarcastic tweets detection based on sentiment hashtags analysis
}

\begin{abstract}
Determining sarcasm among social media is one of the propounded problems from early days. Since recognizing sarcasm is important for development of sentiment analysis systems, identifying sarcastic tweets becomes an issue in this article. Due to the intentional ambiguity in sarcasm, few works have been done in sarcasm detection. In this article, a new Sarcastic Tweets Detection (STD) method is presented for identifying sarcastic tweets more accurate at the level of hashtag. In the proposed STD, Sarcasm Hashtags Classifier (SHC) is developed for classifying tweets into sarcasm and non-sarcasm based on the sentiment analysis of the hashtags. The SHC, works based on the Sarcasm Hashtags Indicator (SHI) and contrast between the orientation of the tweets and hashtag(s). The proposed classifier (SHC) helps us to interpret sarcastic better than the existing work by covering several types of tweets.
\end{abstract}

Keyword: Hashtags sentiment analysis; Sarcastic detection; Sentiment analysis 